\title{
The Effect of Hydrophobicity on Hydraulic Resistance during Transportation of Fluid Media
}

\author{
A.V. Ryzhenkov, M.R. Dasaev, S.V. Grigoriev, A.V. Kurshakov, O.V. Ryzhenkov, M.V. Lukin \\ National Research University "Moscow Power Engineering Institute", Krasnokazarmennaya Str., 17, Moscow, \\ 111250, Russia
}

\begin{abstract}
This work discusses the influence of hydrophobization of functional surfaces on hydraulic resistance during transportation of fluid media. It has been mentioned that the decrease in hydrodynamic loss is stipulated by slip occurring mainly for superhydrophobic surfaces with steady heterogeneous wetting mode.

Pipe samples were made of brass (L63) for experimental studies, their surfaces were modified by various methods: by application of surfactants (contact angle: $111.4^{\circ}$ ) and by formation of micro/nanoscale relief using laser equipment (contact angle: $\sim 156^{\circ}$ ).

Analysis of the experimental data demonstrated that decrease in hydraulic resistance for surfaces modified by surfactants was $5.2 \%$, and for superhydrophobic surfaces modified by laser equipment the hydraulic resistance increased by $8.9 \%$..
\end{abstract}

Key words: drag reduction, hydrophobicity, surface, relief, slip, surfactants, laser textured surface.

\section{INTRODUCTION}

The most common method of long-distance transportation of fluid media is pipeline transport based on conveyance of fluid medium from source to consumer under the action of pressure difference in pipeline cross section created by compressors.

Russia occupies one of the leading positions in the field of pipeline transportation of hydrocarbons, heat carriers, aqueous and other working fluids. For instance, pipeline systems of public heating, water supply, and sewage are inherent features for cities and apartment buildings. The length of pipeline networks in internal utility systems reaches 3-5 million $\mathrm{km} \mathrm{[1].} \mathrm{Herewith,} \mathrm{total} \mathrm{length} \mathrm{of} \mathrm{pipelines} \mathrm{of}$ Russian heating system, one of the most extensive and extended networks in the world, according to various estimations, equals from 20 to 350 thousand $\mathrm{km}[2,3]$. Such significant scopes of supplying systems are stipulated by huge area of the country, its main portion is located in climatic zine with dominating low temperatures for sufficiently long time. The state of such systems is characterized by high wear, low efficiency and reliability resulting from such negative factors as corrosion and erosion, sediment accumulation on functional surfaces. In the course of routine operation, these processes run simultaneously, which increases their influence. Thus, corrosion on functional pipe surfaces, on the one hand, increases roughness increasing the coefficient of hydraulic friction, and on the other hand, accelerates sediment accumulation narrowing pipeline flow area. This leads to increase in hydraulic loss and necessity of regular increase in input pressure of conveyed medium in order to maintain design flow rate, which increases power consumption for transportation and pipe breakages at high working pressure.

While analyzing up-to-date publications, it has been revealed that a challenging approach to improve efficiency of metal pipelines for transportation of aqueous media is based on hydrophobization of functional surfaces. Hydrophobic metal surfaces are characterized by a set of unique properties, including self-cleaning [4], higher corrosion resistance [5], and their application in various fields promotes decrease in hydraulic resistance for transportation of fluid media [6-7], reduced rate of ice formation [8-10], intensification of heat exchange [11] and others.

\section{SLIP OVER HYDROPHOBIC SURFACES}

Increasing number of studies in recent decades devoted to the essence of hydrophobic properties, development of methods of achievement of hydrophobic state of various surfaces, determination of efficiency of hydrophobic surfaces in various scopes demonstrated that the most promising were superhydrophobic surfaces with contact angle higher than $150^{\circ}$.

\subsection{Wetting of hydrophobic surfaces}

It has been mentioned in the previous work [12] that the maximum contact angle upon hydrophobization of smooth surface by modification of chemical composition of surface layer does not exceed $120^{\circ}$. Achievement of higher values is provided by roughness which also exerts significant influence on thermal and hydrodynamic state of fluid droplet on surface. Superhydrophobic surfaces are characterized by homogeneous (the Wenzel state [13]) and heterogeneous (the Cassie-Baxter state [14, 15]) wetting modes. Herewith, 
wetting mode on superhydrophobic surface will be determined by surface relief geometry [16].

The homogeneous wetting, when fluid contacts with overall surface filling all cavities, is characterized by high adhesion at fluid/solid interface. Despite high contact angle, a water droplet adheres to superhydrophobic surface and does not evacuate at any tilt angles. Such surfaces can be exemplified well by rose petal surfaces and gecko adherence system [17, 18]. Such surfaces are widely applied, for instance, upon transportation of microdroplets of supermagnetic fluid using magnetic fields [19, 20].

However, the heterogeneous wetting is characterized by low adhesion at fluid/solid interface resulting in low angle of fluid droplet roll-off from surface. In this case the fluid contacts only with protrusions on surface, whereas pores and cavities contain air presenting contact with aqueous medium. This can be exemplified well by such surface as lotus leaf characterized by self-cleaning due to water droplet roll-off from surface [19, 21]. In addition, numerous insects and animals are characterized both by superhydrophobic and self-cleaning properties and by decrease in resistance in fluid flows [22].

Analysis of various states of superhydrophobic surfaces has demonstrated that, aiming at efficiency improvement of pipelines for transportation of aqueous medium, the optimum results will be obtained on the basis of superhydrophobic surfaces with heterogeneous wetting.

\subsection{Reduction of hydraulic resistance}

The slip distance $b$ is equivalent to the distance where the velocity profile is extrapolated to zero (Fig. 1, b) and is determined by the ratio of fluid slip velocity on surface $u_{z}$ to the respective shear rate on surface $\frac{\partial u}{\partial s}$ :

$$
b=\frac{u_{z}}{\partial u / \partial z}
$$

The shear stress on solid surface $\sigma_{g}$ is proportional to the slip velocity $u_{s}$, and the proportionality factor is the friction coefficient $\varepsilon$ :

$$
\sigma_{s}=s u_{s}=\eta \frac{\partial u}{\partial z}
$$

where $\eta$ is the viscosity of fluid. Based on Eqs. (1) and (2), for the slip length $b$ it is possible to write as follows:

$$
b=\frac{\eta}{\varepsilon}
$$

At the slip distance $b=0$ (Fig. 1, a), the boundary condition is reduced to classical condition of no-slip (adherence) of fluid on surface and corresponds to the velocity $u_{s}=0$. The slip length $b \rightarrow \infty$ is determined by no-friction $\varepsilon \rightarrow 0$ and takes place in the case of ideal slip characteristic for plug flow at zero velocity gradient $\frac{\partial_{u}}{\partial g}=0$

(Fig. 1, c) [24]. In addition, the slip length $b$, as illustrated by Eqs. (1) and (3), depends on the parameters determining properties of surface (friction coefficient ${ }^{\varepsilon}$ ) and fluid (viscosity $\eta$ ).

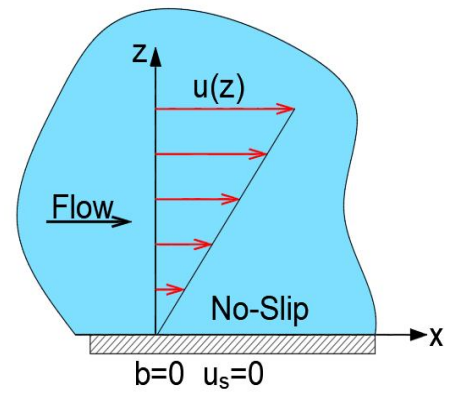

(a)

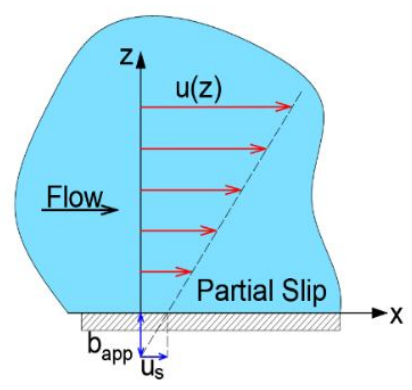

(b)

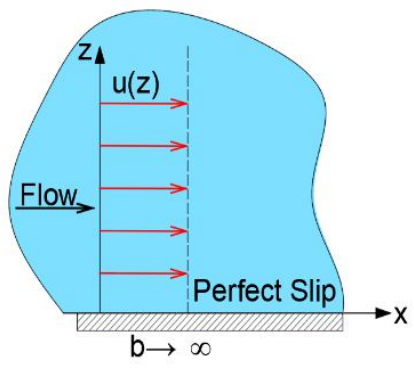

(c)

Figure 1: Schematic view of velocity profile and slip distance upon fluid flow over solid surface under the following boundary conditions: (a) no-slip (adherence); (b) partial slip; and (c) perfect slip (no-friction).

In recent years it has been recognized that unique properties of superhydrophobic surfaces caused by relief geometry can influence significantly fluid dynamics near solid surface and, as a consequence, promote decrease in hydraulic resistance upon transportation of fluid media. This is stipulated by fluid hydrodynamic slip near solid superhydrophobic surface $[25$,
26].

At present the following slip models are determined:

- real (molecular) slip (Fig. 2, a);

- apparent (observed) slip (Fig. 2, b);

- effective slip (Fig. 2, c). 

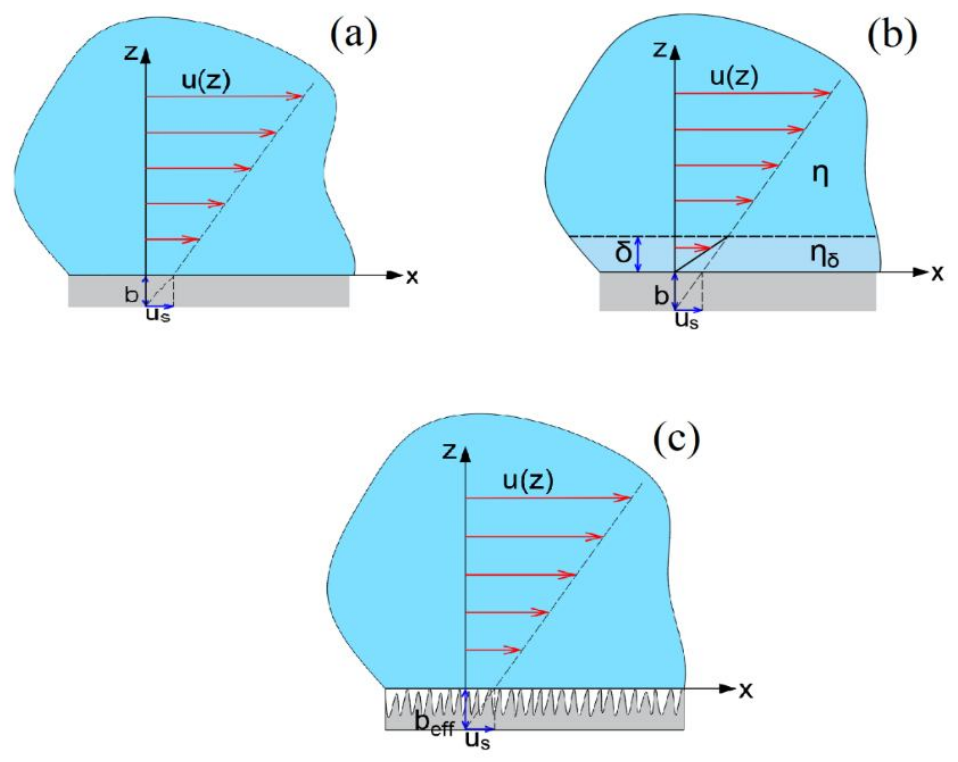

Figure 2: Schematic view of models of (a) real, (b) apparent, and (c) effective slip.

Computer molecular dynamic simulations have demonstrated that slipping of molecules directly above solid surface can be caused by the fact that cohesion of fluid molecules exceeds adhesion at solid/fluid interface, that is, on hydrophobic surface [27-29]. However, the simulated slip distances $b$ were low (not more than $10 \mathrm{~nm}$ ) [30, 31].

In some tests with hydrophobic surfaces the slip distance reached up to $\sim 100 \mathrm{~nm}[30,32]$, which exceeded significantly the simulated results of molecular slip. In this regard the model of apparent (observed) slip was developed which assumed formation of thin lubricating layer on surface (gaseous or liquid film with significantly lower viscosity in comparison with that in bulk $\eta_{\delta} \ll \eta$ ) with the thickness $\delta$ (Fig. 2, b) [24, 32]. The apparent slip is provided due to higher shear rate $\partial u / \partial z$ in low viscosity $\delta$ layer in comparison with bulk shear rate. The distance of apparent slip is determined as follows [24]:

$$
b_{a p p}=\delta\left(\frac{\eta}{\eta_{\tilde{k}}}-1\right) \approx \delta \frac{\eta}{\eta_{\tilde{k}}}
$$

Despite its seeming simplicity, this slip model reflects formation of low viscosity layer near hydrophobic wall and allows to understand the influence of interface structure on interfacial transportation phenomena.

In the case of slip over heterogeneous surface, it would be reasonable to consider the effective slip distance $b_{\text {eff }}$ (Fig. 2, c) instead of local system description, that is, averaged with respect to characteristic scale of heterogeneity [33-35]. Such approach allows to determine solutions to the problems and to analyze complex systems without cumbersome computations of local field of fluid velocity. Herewith, the effective slip distance $b_{\text {off }}$ is determined by the ratio of average slip velocity $\left\langle u_{s}\right\rangle$ to average shear rate near wall:

$$
b_{\text {eff }}=\frac{\left\langle u_{s}\right\rangle}{\langle\partial u / \partial z\rangle} .
$$

The use of effective slip distance $b_{\text {eff }}$ makes it possible to substitute actual heterogeneous surface with smooth plane with the same observed properties as the actual surface.

It has been demonstrated in $[7,36]$ that superhydrophobic surfaces allow to increase the slip distance by several hundred of microns by means of steady gaseous phase constrained in the cavities of microrelief. Therefore, they are highly attractive for reduction of hydrodynamic resistance.

\section{EXPERIMENTAL}

\subsection{Test rig}

Aiming at determination of the influence of superhydrophobic surfaces on hydraulic resistance upon transportation of aqueous medium, the test rig was developed (Fig. 3).

Water circulates over closed circuit comprised of the test section, the tank, the water flow meter, and the circulation pump. The tank is preliminary filled with cold tap water. The pressure drop at the test section is detected using liquid U-shaped differential pressure meter. 


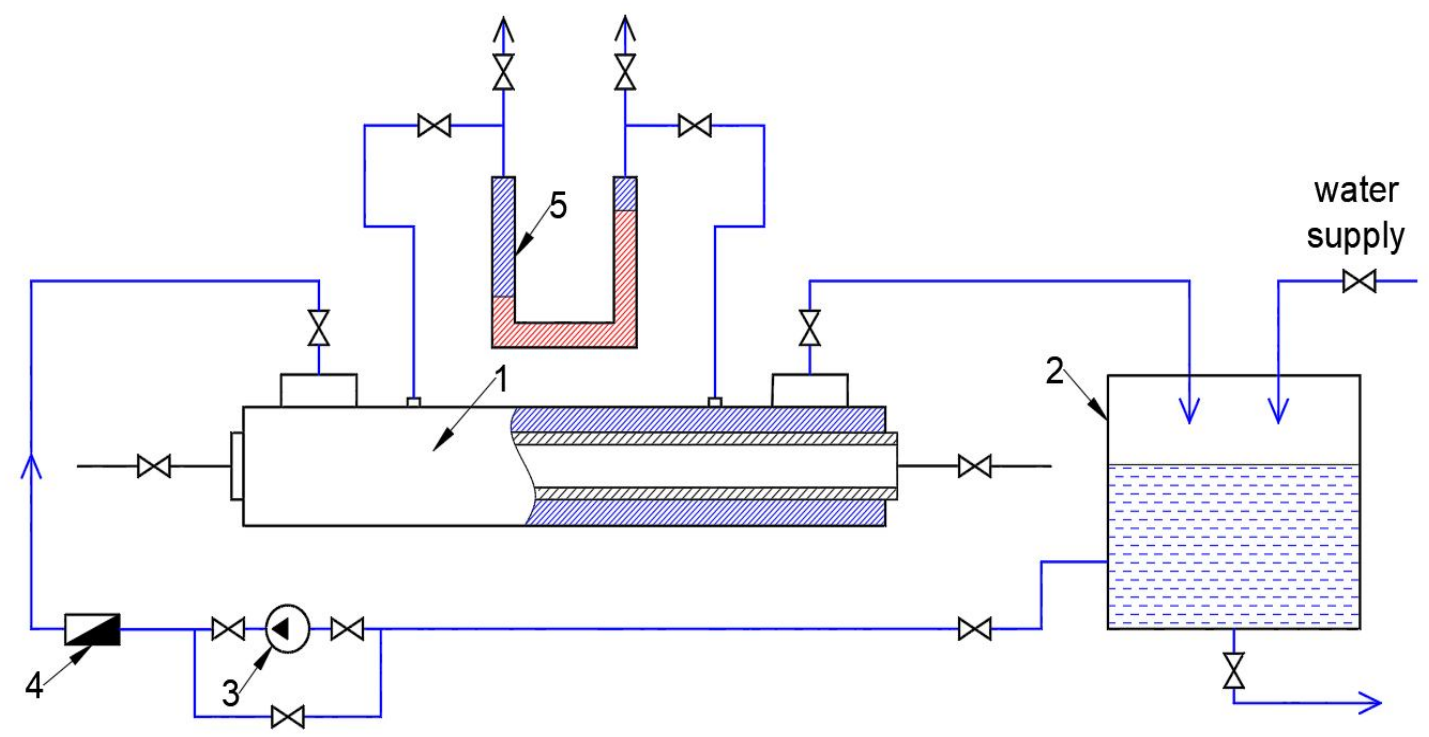

Figure 3: Schematic view of test rig: 1 - test section, 2 - tank, 3 - circulation pump, 4 - water flow meter, 5 - U-shaped pressure meter.

The test section (Fig. 4) for water flow is comprised of annular space arranged by external pipe with the outer diameter of 90 $\mathrm{mm}$ with the wall thickness of $3 \mathrm{~mm}$ and internal pipe with the outer diameter of $30 \mathrm{~mm}$ and the wall thickness of $1 \mathrm{~mm}$.
The internal pipe is made of brass, grade L63, and the external pipe is made of steel, grade St20. The overall section distance is $2,000 \mathrm{~mm}$.
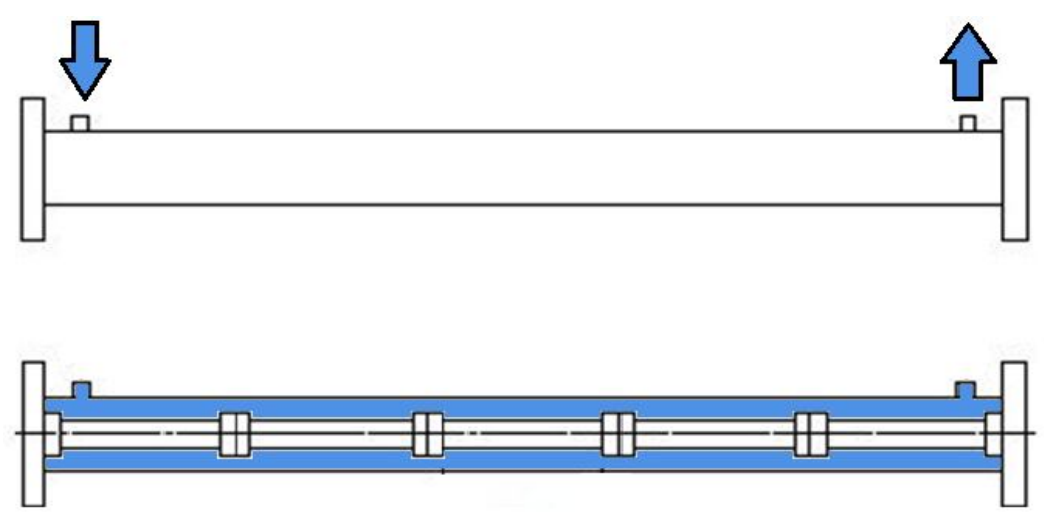

Figure 4: Schematic view of test section.

Due to restrictions related with the maximum allowable length of a sample which can be installed into the rotational unit upon laser modification of surface, the internal pipe is subdivided into five elements, the length of each is $400 \mathrm{~mm}$. It should be mentioned that treatment of internal pipe surfaces attracts great attention for reduction of hydraulic resistance. In this work the internal pipe surface could not be treated, and only external pipe surface was treated.

\subsection{Production and modification of surface of test samples}

In the course of tests, the samples with various surfaces were used including:

- initial surfaces;

- surfaces modified by surfactants;

- surfaces modified by laser equipment.

As-delivered pipes were used as samples with initial surfaces.
The measured contact angle on such surfaces was $78.3^{\circ}$.

\subsection{Surface modification by surfactants}

The functional surface of pipe samples was modified by the following surfactant: octadecylamine, a film forming aliphatic amine (ODA).

The ODA molecular layers were formed on external surfaces of test samples by means of special separate circuit comprised of the process tank, the circulation pump, and the ejecting unit including the metering tank for surfactants, the shut-off adjusting valves, the connecting pipelines and the ejector. This circuit provides preparation, circulation, heating and maintaining preset temperature of emulsion or solution to which samples are immersed during the required time of formation of molecular layers on the treated surfaces.

The contact angle measured after the described modification 
of brass surfaces was $111.4^{\circ}$.

\subsection{Surface modification by laser}

The modification of surfaces by laser equipment was based on formation of micro/nanoscale relief using laser pulse impact applied to the surface. Similar studies aimed at achievement of hydrophobic state of brass surfaces were described in $[12$, 37].

The relief was formed during passing of laser beam over surface of rotating pipe so that the increment between two generated spiral turns was $100 \mu \mathrm{m}$. The parameters of laser radiation upon the modification were as follows:

- laser radiation power: $20 \mathrm{~W}$;

- laser radiation frequency: $20 \mathrm{kHz}$;

- linear velocity of beam travel along pipe surface: $100 \mathrm{~mm} / \mathrm{s}$. After holding the test samples with laser modified surfaces in air under standard ambient conditions in 14 days, the wetting parameters were analyzed. The contact angles were measured by placing droplets of various volumes onto the surface: 2,4 , and $5 \mu \mathrm{l}$. Different droplet volumes were stipulated by complicated studies caused by sufficiently low roll-off angle and surface curvature of samples.

It has been determined that the droplet volume effects the contact angle of surfaces. Herewith, the contact angle decreases with the increase in droplet volume (Fig. 5).

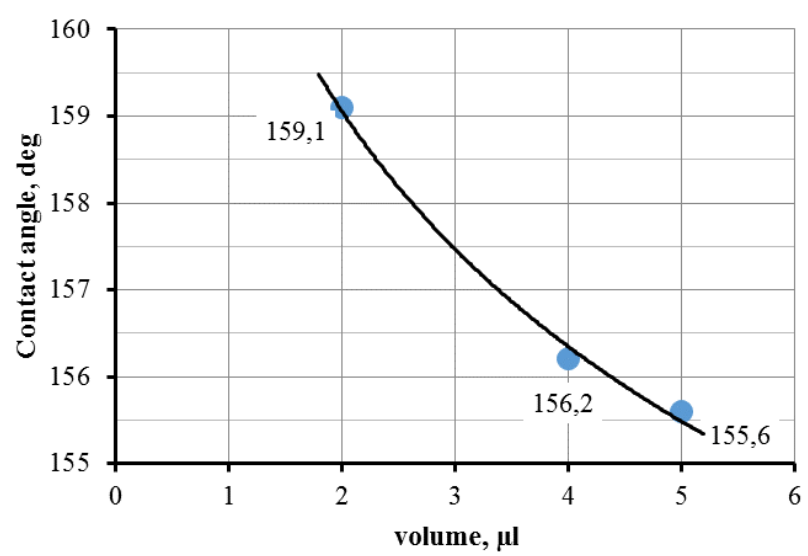

Figure 5: Contact angle on hydrophobic surface as a function of droplet volume

As can be seen in Fig. 6, where droplets of various volume (2, 4 , and $5 \mu \mathrm{l}$ ) on surface modified by laser equipment are shown, heterogeneous wetting can be observed.

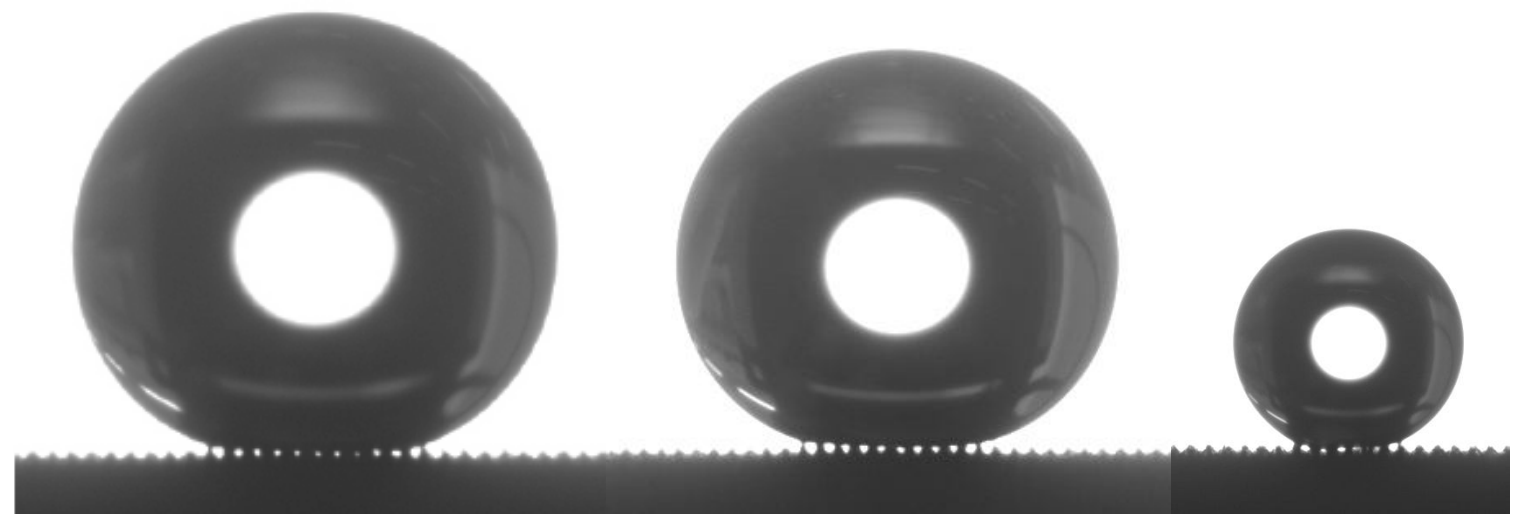

Figure 6: Photos of droplets with the volumes of 5, 4, and $2 \mu \mathrm{l}$ (from left to right) on modified surface in equal scale.

\section{RESULTS AND DISCUSSION}

Based on experimental data, the hydraulic resistance along the test section was plotted as a function of Re number (Fig. 7 ); it can be seen that the loss was decreased only for experimental samples modified by surfactants. Herewith, the maximum effect is observed under conditions of turbulent flow of aqueous medium at $\mathrm{Re}>10,000$ and the maximum relative decrease is $5.2 \%$ at $\operatorname{Re}=15,000$.

For the test samples with surfaces modified by laser equipment, the hydraulic resistance increased by $8.9 \%$ at $\operatorname{Re}=15,000$. 
A.V. Ryzhenkov et al., International Journal of Emerging Trends in Engineering Research, 8(1), January 2020, 195 - 202

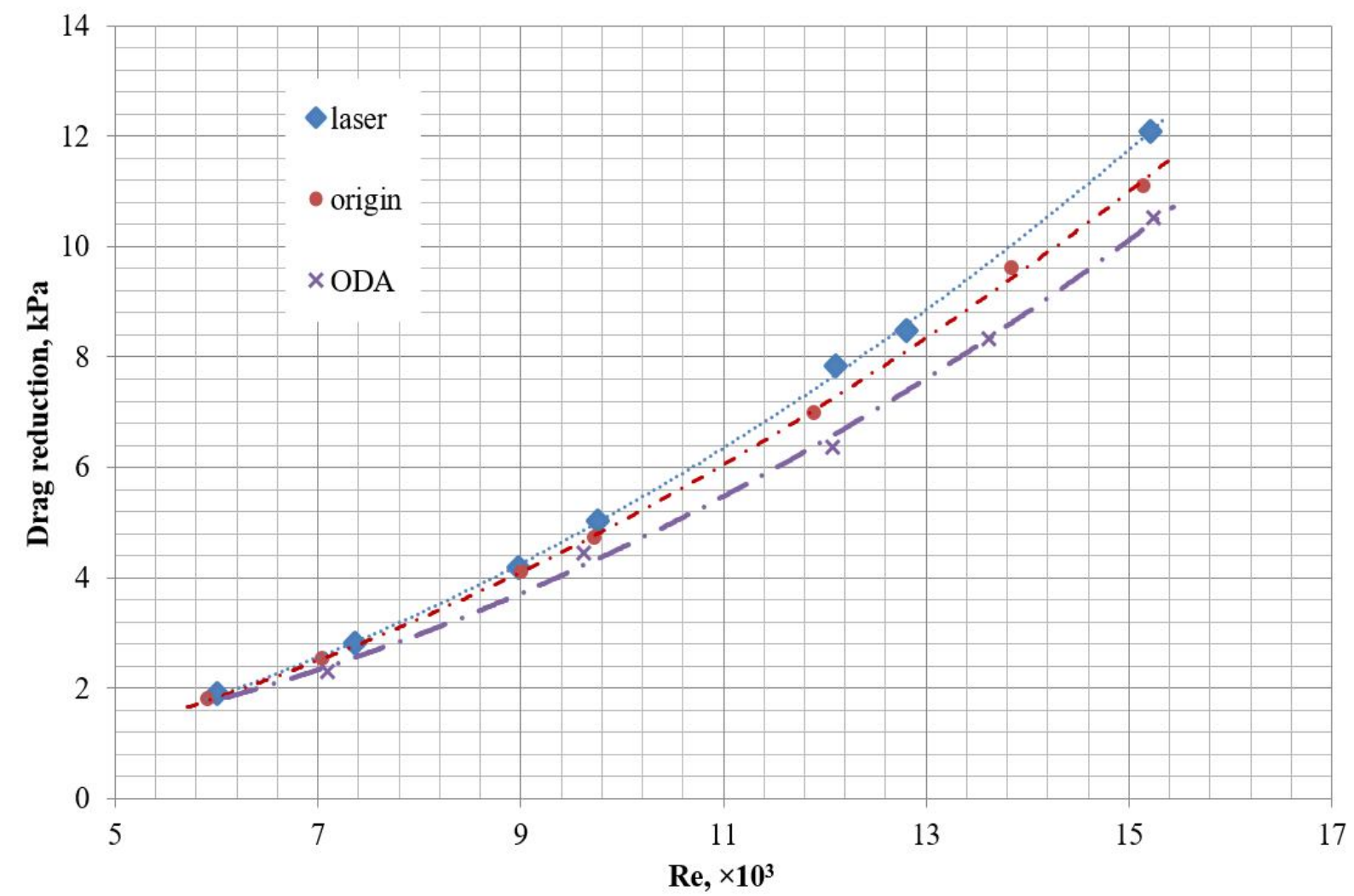

Figure 7: Hydraulic resistance as a function of Re number for test samples: 'laser' - surface modification by laser equipment, 'original' - initial surface, 'ODA' - surface modification by surfactants.

It should be mentioned that immediately after the tests, the internal pipe with laser modified surface was dismounted, its surface was wetted with water, i.e. was characterized by hydrophilic properties. This stipulates the increase in hydraulic loss and could be attributed to the following factors: - superhydrophobic state was achieved as a consequence of relief formation on laser modified surface and decrease in surface energy due to physically adsorbed organic layer [12]. However, such layer can be easily removed from the surface as a consequence of interaction with fluid medium, which initiates uncontrolled loss of superhydrophobic state [38].

- in the case of complex reliefs, fluid can wet surface partially, which corresponds to metastable state under which a water droplet on surface is characterized by the state intermediate between heterogeneous and homogeneous wetting [39]. Transition from metastable heterogeneous wetting to steady homogeneous mode can be caused by the impact of various impacts, including vibration [40], evaporation [41, 42], air diffusion [43, 44], or droplet falling [45, 46].

\section{CONCLUSION}

It has been revealed that application of hydrophobic surfaces decreases hydraulic resistance upon transportation of fluid media.

It has been mentioned that the maximum decrease in hydraulic resistance for the surfaces modified by surfactants is observed upon turbulent flow of aqueous medium equaling to
$5.2 \%$ at $\boldsymbol{R e}=15,000$. For superhydrophobic surfaces modified by laser equipment, the hydraulic resistance increased by $8.9 \%$ at $R e=15,000$.

It is assumed that the decrease in hydraulic resistance is stipulated by loss of superhydrophobic properties of surfaces due to such factors as removal of organic layer upon contact with aqueous medium and/or as consequence of external factors, for instance, excessive pressure of aqueous medium. The latter factor leads to unsuitability of such coating for commercial application, especially externally. However, for instance, octadecylamine, would probably lead to formation of solid and chemically stable superhydrophobic coatings. It should be mentioned that knowledge of transition from heterogeneous to homogeneous wetting and dynamics of metastable state is important for control and improvement of superhydrophobic surfaces.

\section{REFERENCES}

1. V.N. Isaev, R.Yu. Khurgin. Truboprovodnye kommunal'nye sistemy [Pipeline utility systems]. Santekhnika, Vol. 3, pp. 52-60, 2006.

2. I.A. Bashmakov, A.D. Myshak. Rossiiskaya sistema ucheta povysheniya energoeffektivnosti i ekonomii energii [Russian accounting system of energy efficiency improvement and saving]. Moscow, TsENEF, 2012. 
A.V. Ryzhenkov et al., International Journal of Emerging Trends in Engineering Research, 8(1), January 2020, 195 - 202

3. V.M. Lipovskikh. Osnovnye napravleniya energoeffektivnosti pri ekspluatatsii teplovykh setei [Main trends of energy efficiency upon operation of heating services]. Energosberezhenie, Vol. 1, 10-13, 1999.

4. H.G., Yilbas B.S., Al-Sharafi A. Self-cleaning of a hydrophobic surface by a rolling water droplet. Sci Rep., Vol. 9., pp. 5744, 2019. https://doi.org/10.1038/s41598-019-42318-3

5. S. Pan, A.K. Kota, J.M. Mabry, A. Tuteja. Superomniphobic surfaces for effective chemical shielding. J. Am. Chem. Soc., Vol. 135, pp. 578-581, 2013.

6. G. McHale, M.I. Newton, N.J. Shirtcliffe. Immersed superhydrophobic surfaces: Gas exchange, slip and drag reduction properties. Soft Matter, Vol. 6, pp. 714-719, 2010.

7. C. Lee, C.J. Kim. Underwater restoration and retention of gases on superhydrophobic surfaces for drag reduction. Phys. Rev. Lett., Vol. 106, 014502, 2011. https://doi.org/10.1103/PhysRevLett.106.014502

8. R. Menini, Z. Ghalmi, Farzaneh M. Highly resistant icephobic coatings on aluminum alloys. Cold Reg. Sci. Technol., Vol. 65, pp. 65-69, 2011.

9. P. Kim, T.S. Wong, J. Alvarenga, M.J. Kreder, W.E. Adorno-Martinez, J. Aizenberg. Liquid-Infused Nanostructured Surfaces with Extreme Anti-Ice and Anti-Frost Performance. ACS Nano, Vol. 6, pp. 6569-6577, 2012.

10. L.B. Boinovich, A.M. Emelyanenko. Anti-icing potential of superhydrophobic coatings. Mendeleev Communications, Vol. 23, pp. 3-10, 2013.

11. O.V. Ryzhenkov, A.V. Kurshakov, A.V. Ryzhenkov, M.R. Dasaev, S.V. Grigoriev. On intensification of heat exchange in steam condensers made of stainless steel and brass. International Journal of Innovative Technology and Exploring Engineering, Vol. 8, pp. 2290-2294, 2019.

12. A.V. Ryzhenkov, M.R. Dasaev, S.V. Grigoriev, A.V. Kurshakov, O.V. Ryzhenkov, M.V. Lukin. Hydrophobic brass surfaces created by means of multi-scale relief. International Journal of Mechanical Engineering and Technology, Vol. 9, pp. 58-70, 2018.

13. R.N. Wenzel. Resistance of solid surfaces to wetting by water. Ind. Eng. Chem., Vol. 28(8), pp. 988- 994, 1936. https://doi.org/10.1021/ie50320a024

14. A.B.D. Cassie, S. Baxter. Large contact angles of plant and animal surfaces. Nature. 1945. Vol. 155. P. 21-22.

15. S. Baxter, A.B.D. Cassie. 8-The water repellency of fabrics and a new water repellency test. Journal of the Textile Institute Transactions, Vol. 36, No. 4, pp. T67-T90, 1945.

16. A. Marmur. Wetting on Hydrophobic Rough Surfaces: To Be Heterogeneous or Not To Be? Langmuir, Vol. 19(20), pp. 8343-8348, 2003.

17. D. Eber, B. Bhushan. Wear-resistant rose petal-effect surfaces with superhydrophobicity and high droplet adhesion using hydrophobic and hydrophilic nanoparticles. J. Colloid Interface Sci., Vol. 384, pp. 182-188, 2012.

18. L. Feng, Y. Zhang, M. Li, Y. Zheng, W. Shen, L. Jiang. The structural color of red Rose petals and their duplicates. Lagmuir, Vol. 26, pp. 14885-14888, 2010.

19. L. Feng, Y. Zhang, J. Xi, Y. Zhu, N. Wang, F. Xia, L. Jiang. Petal effect: A superhydrophobic state with high adhesive force. Langmuir, Vol. 24, pp. 4414-4419, 2008. https://doi.org/10.1021/la703821h

20. S. Nishimoto, B. Bhushan. Bioinspired self-cleaning surfaces with superhydrophobicity, superoleophobicity, and superhydrophilicity. RSC Adv., Vol. 3, pp. 671-690, 2013.

21. T. Sun, L. Feng, X. Gao, L. Jiang. Bioinspired surfaces with special wettability. Acc. Chem. Res., Vol. 38(8), pp. 644-652, 2005.

22. Z. Pan, F. Cheng, B. Zhao. Bio-Inspired Polymeric Structures with Special Wettability and Their Applications: An Overview. Polymers., Vol. 9(12), p. 725, 2017.

23. C.L.M.H. Navier. Mémoire sur les lois du Mouvement des Fluides. Mémoires de l'Académie Royale des Sciences de l'Institut de France, pp. 389-440, 1823.

24. O.I. Vinogradova. Drainage of a thin liquid film confined between hydrophobic surfaces. Langmuir, Vol. 11, pp. 2213-2220, 1995. https://doi.org/10.1021/la00006a059

25. J.P. Rothstein. Slip on superhydrophobic surfaces. Annual Review of Fluid Mechanics, Vol. 42(1), pp. 89-109, 2010.

26. R.S. Voronov, D.V. Papavassiliou, L.L. Lee. Review of fluid slip over superhydrophobic surfaces and its dependence on the contact angle. Ind. Eng. Chem. Res., Vol. 47., pp. 2455-2477, 2008.

27. P. Thompson, S. Troian. A general boundary condition for liquid flow at solid surfaces. Nature, Vol. 389, pp. 360-362, 1997.

28. J.-L. Barrat, L. Bocquet. Influence of wetting properties on hydrodynamic boundary conditions at a fluid/solid interface. Faraday Discuss, Vol. 112, p. 119-128, 1999. https://doi.org/10.1039/a809733j

29. M. Sun, C. Ebner. Molecular dynamics study of flow at a fluid-wall interface. Phys. Rev. Lett., Vol. 69, P. 3491, 1992.

30. D. Huang, C. Sendner, D. Horinek, R. Netz, L. Bocquet. Water Slippage versus Contact Angle: A Quasiuniversal Relationship. Phys. Rev. Lett., Vol. 101, p. 226101, 2008.

31. C. Sendner, D. Horinek, L. Bocquet, R. Netz. Interfacial Water at Hydrophobic and Hydrophilic Surfaces: Slip, Viscosity, and Diffusion. Langmuir, Vol. 25, pp. 10768-10781, 2009.

32. D. Andrienko, B. Dünweg, O.I. Vinogradova. Boundary slip as a result of a prewetting transition. The Journal of Chemical Physics, 119(24), pp. 13106-13112, 2003. 
33. C.H. Choi, U. Ulmanella, J. Kim, C.M. Ho, C.J. Kim. Effective slip and friction reduction in nanograted superhydrophobic microchannels. Phys Fluids, Vol. 18, 087105, 2006.

https://doi.org/10.1063/1.2337669

34. K. Kamrin, M. Bazant, H. Stone. Effective slip boundary conditions for arbitrary periodic surfaces: The surface mobility tensor. Journal of Fluid Mechanics, Vol. 658, pp. 409-437, 2010.

35. F. Feuillebois, M.Z. Bazant, O.I. Vinogradova. Effective slip over superhydrophobic surfaces in thin channels. Phys. Rev. Lett., Vol. 102, pp. 1-5, 2009.

36. C. Lee, C.J. Kim. Maximizing the Giant Liquid Slip on Superhydrophobic Microstructures by Nanostructuring Their Sidewalls. Langmuir, Vol. 25, No. 21, pp. 1281212818, 2009.

37. A.V. Ryzhenkov, M.R. Dasaev, A.V. Kurshakov, O.V. Ryzhenkov, S.V. Grigoriev and M.V. Lukin, Production of Superhydrophobous Surfaces Using Laser Texturing. International Journal of Mechanical Engineering and Technology, Vol. 8(11), pp. 746-755, 2017.

38. L.B. Boinovich, A.M. Emelyanenko, K.A. Emelyanenko, A.G. Domantovsky, A.A. Shiryaev. Comment on "Nanosecond laser textured superhydrophobic metallic surfaces and their chemical sensing applications" by V.Ta Duong, A. Dunn, T.J. Wasley, R.W. Kay, J. Stringer, P.J. Smith, C. Connaughton, J.D. Shephard (Appl. Surf. Sci., Vol. 357, pp. 248-254, 2015). Appl. Surf. Sci., Vol. 379, pp. 111-113, 2016.

39. H.M. Kwon, A.T. Paxson, K.K. Varanasi, N.A. Patankar. Rapid Deceleration-Driven Wetting Transition during Pendant Drop Deposition on Superhydrophobic Surfaces. Phys. Rev. Lett., Vol. 106: 036102, 2011.

40. E. Bormashenko, R. Pogreb, G. Whyman, M. Erlich. Cassie-Wenzel Wetting Transition in Vibrating Drops Deposited on Rough Surfaces: Is the Dynamic Cassie-Wenzel Wetting Transition a 2D or 1D Affair? Langmuir., Vol. 23(12). P. 6501-6503, 2007. https://doi.org/10.1021/la700935x

41. M. Reyssat, J. M. Yeomans, D. Quéré. Impalement of fakir drops. Europhys. Lett., Vol. 81:26006, 2008.

42. P. Tsai, R.G.H. Lammertink, M. Wessling, D. Lohse. Evaporation-Triggered Wetting Transition for Water Droplets upon Hydrophobic Microstructures. Phys. Rev. Lett., Vol. 104: 116102, 2010.

43. M.S. Bobji, S.V. Kumar, A. Asthana, R.N. Govardhan. Underwater Sustainability of the "Cassie" State of Wetting. Langmuir, Vol. 25(20), pp. 12120-12126, 2009.

44. M.A. Samaha, F.O. Ochanda, H.V. Tafreshi, Gary C. Tepper, M. Gad-el-Hak. In situ, noninvasive characterization of superhydrophobic coatings. Review of Scientific Instruments. 2011. Vol. 82:045109.

45. H.M. Kwon, A.T. Paxson, K.K. Varanasi, N.A. Patankar. Rapid Deceleration-Driven Wetting Transition during Pendant Drop Deposition on Superhydrophobic Surfaces. Phys. Rev. Lett., Vol. 106: 036102, 2011.
46. T. Deng, K.K. Varanasi, M. Hsu, N. Bhate, C. Keimel, J. Stein, M. Blohm. Nonwetting of impinging droplets on textured surfaces. Appl. Phys. Lett., Vol. 94(13), 133109, 2009.

https://doi.org/10.1063/1.3110054 